Portland State University

PDXScholar

$11-1-2012$

\title{
Self-assembled nanoparticle antiglare coatings
}

\author{
Khalid Askar \\ University of Florida \\ Blayne M. Phillips \\ University of Florida \\ Xuan Dou \\ University of Florida \\ Juan Lopez \\ University of Florida \\ Carl Smith \\ University of Florida
}

See next page for additional authors

Follow this and additional works at: https://pdxscholar.library.pdx.edu/mth_fac

Part of the Mathematics Commons

Let us know how access to this document benefits you.

\section{Citation Details}

Jiang, B., Askar, K., Phillips, B., Dou, X., Lopez, J., Smith, C., and Jiang, P. (2012). Self-assembled nanoparticle antiglare coatings. Vol. 37 (21), p. 4380-4382

This Article is brought to you for free and open access. It has been accepted for inclusion in Mathematics and Statistics Faculty Publications and Presentations by an authorized administrator of PDXScholar. Please contact us if we can make this document more accessible: pdxscholar@pdx.edu. 
Authors

Khalid Askar, Blayne M. Phillips, Xuan Dou, Juan Lopez, Carl Smith, Bin Jiang, and Peng Jiang 


\title{
Self-assembled nanoparticle antiglare coatings
}

\author{
Khalid Askar, ${ }^{1}$ Blayne M. Phillips, ${ }^{1}$ Xuan Dou, ${ }^{1}$ Juan Lopez, ${ }^{1}$ Carl Smith,,${ }^{1}$ Bin Jiang, ${ }^{2}$ and Peng Jiang ${ }^{1, *}$ \\ ${ }^{1}$ Department of Chemical Engineering, University of Florida, Gainesville, Florida 32611, USA \\ ${ }^{2}$ Fariborz Maseeh Department of Mathematics and Statistics, Portland State University, Portland, Oregon 97201, USA \\ ${ }^{*}$ Corresponding author: pjiang@che.ufl.edu
}

Received August 3, 2012; revised September 13, 2012; accepted September 14, 2012; posted September 17, 2012 (Doc. ID 173845); published October 17, 2012

\begin{abstract}
Here we report a simple and scalable bottom-up technology for assembling close-packed nanoparticle monolayers on both sides of a glass substrate as high-quality antiglare coatings. Optical measurements show that monolayer coatings consisting of $110 \mathrm{~nm}$ silica nanoparticles can significantly reduce optical reflectance and enhance specular transmittance of the glass substrate for a broad range of visible wavelengths. Both experiments and numerical simulations reveal that the antiglare properties of the self-assembled colloidal monolayers are significantly affected by the size of the colloidal particles. (C) 2012 Optical Society of America
\end{abstract}

OCIS codes: $240.0310,310.1210$.

Light reflection from an optical surface, such as automotive dashboards and flat panel displays, could pose safety hazards, impair the legibility of displays, and degrade performance of optical systems with multiple optical components [1,2]. Glass with a typical refractive index of $\sim 1.5$ reflects $\sim 4 \%$ of normal incident light from each air-glass interface. Traditional quarter-wavelength antiglare or antireflection coatings are widely used on glass surface to reduce light reflection and increase optical transmission [3]. However, depositing quarter-wavelength coatings usually requires high vacuum conditions, impeding cost effectiveness of the fabrication processes.

A large variety of antiglare/antireflection technologies based on simple solution processing have been extensively developed [4]. For instance, nanoporous coatings created by phase separation of polymer blends, selfassembly of nanoparticle and polyelectrolyte multilayers, and many other approaches are widely used in fabricating antireflection coatings on various substrates [5-7]. Subwavelength-structured moth-eye coatings made by both top-down and bottom-up techniques can reduce light reflection over a broad range of wavelengths and angles of incidence [몰.

Nanoparticle self-assembly provides a simple and inexpensive approach in fabricating antiglare/antireflection coatings. Monolayers of colloidal nanoparticles created by convective assembly or spin casting have been utilized as antireflection coatings on crystalline silicon solar cells and glass substrates [10-13]. Layer-by-layer (LBL) assembly of nanoparticles and polyelectrolytes enables highperformance antiglare coatings on nonplanar substrates $[\underline{6}, 14]$. Self-assembled colloidal arrays are also widely used as structural templates in fabricating moth-eye antireflection coatings [15-17]. Unfortunately, many of the available bottom-up techniques involve multiple steps (e.g., LBL assembly) and/or are limited to singlesided coatings on planar substrates. Here we report a single-step colloidal self-assembly technology that enables simultaneous deposition of nanoparticle monolayers on both sides of a glass substrate.

Monodispersed silica spheres with diameter of 110 and $210 \mathrm{~nm}$ and size distribution of less than $6 \%$ were synthesized by the standard Stöber method [1]. The as-synthesized silica particles were purified by repeated centrifugation/redispersion cycles in ethanol and were finally redispersed in ethylene glycol with particle volume fraction of 0.20 . Figure 1 shows a schematic illustration of the colloidal self-assembly process. The silica/ ethylene glycol suspension was added dropwise to the surface of deionized water contained in a glass crystallizing dish. The silica spheres were accumulated at the air/water interface. Because of the high surface tension of water $\left(72.75 \mathrm{mN} / \mathrm{m}\right.$ at $\left.20^{\circ} \mathrm{C}\right)$, the strong capillary action between neighboring microspheres can then organize the floating particles into close-packed monolayer colloidal crystals that exhibit iridescent colors caused by light diffraction [17]. A glass microslide $(75 \mathrm{~mm} \times 25 \mathrm{~mm})$ preimmersed in water was vertically withdrawn at a rate of $\sim 12.5 \mathrm{~mm} / \mathrm{min}$ by using a clamp attached to a syringe pump (KD Scientific 780-230). The floating colloidal crystals were evenly transferred onto both sides of the glass substrate.

Figure 2(a) shows a photograph of a glass microslide with the right half covered by a transferred layer of $110 \mathrm{~nm}$ silica spheres. The high uniformity of the coating is apparent from the picture. In addition, by comparing with the bare glass substrate (left half of the microslide), the significant reduction in optical reflection from the self-assembled nanoparticle antiglare coating is clearly evident. By contrast, the photograph in Fig. 2(b) shows that a floating-assembled colloidal crystal consisting of $210 \mathrm{~nm}$ silica spheres (deposited on the right half of

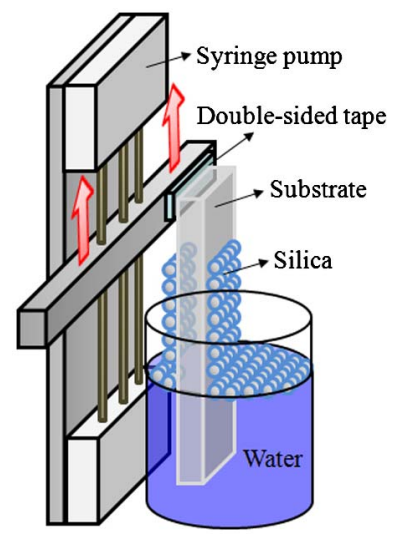

Fig. 1. (Color online) Schematic illustration of the colloidal self-assembly process for depositing colloidal monolayers on both sides of a substrate in a single step. 


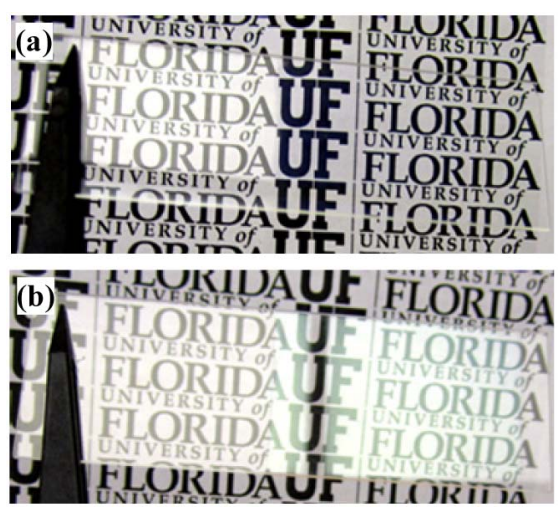

Fig. 2. (Color online) Photographs of glass slides $(75 \mathrm{~mm} \times 25 \mathrm{~mm})$ with the right halves covered by closepacked monolayers of (a) $110 \mathrm{~nm}$ and (b) $210 \mathrm{~nm}$ silica spheres.

the microslide) increases optical reflection more than a bare glass substrate (left half of the substrate).

Figure 3(a) displays a typical top-view scanning electron microscope (SEM) image of the self-assembled $110 \mathrm{~nm}$ silica colloidal crystal as shown in Fig. 2(a). Although a number of defects (e.g., agglomerated particles and grain boundaries) are present, the hexagonal ordering of silica nanoparticles is clearly depicted. Fortunately, these intrinsic defects (many of them are subwavelength scale) do not significantly affect the optical uniformity and the antiglare properties of the coatings. Magnified top-view and cross-sectional SEM images (not shown here) further demonstrate that the floatingassembled colloidal crystals are monolayers. Figure 3(b) shows a top-view SEM image of the assembled $210 \mathrm{~nm}$ colloidal monolayer. The single crystalline domain size is apparently much larger than that of the $110 \mathrm{~nm}$ colloidal coating. This is reasonable as the capillary action between neighboring spheres, which is the major driving force for the observed colloidal crystallization, is proportional to the size of the colloidal spheres [19]. The strong capillary action and the reduced Brownian motion lead to the observed long-range hexagonal ordering for particles with diameter larger than $\sim 200 \mathrm{~nm}$.

The specular optical reflection and transmission measurements of the self-assembled colloidal monolayers were carried out using a HR4000 + NIR512 Vis-NIR spectrometer (Ocean Optics). Figure 4(a) compares the specular reflection spectra obtained at normal incidence from a flat glass substrate and the floating-assembled 110 and $210 \mathrm{~nm}$ colloidal monolayers. The flat glass microslide exhibits a double-side reflectance of $\sim 8.5 \%$, agreeing
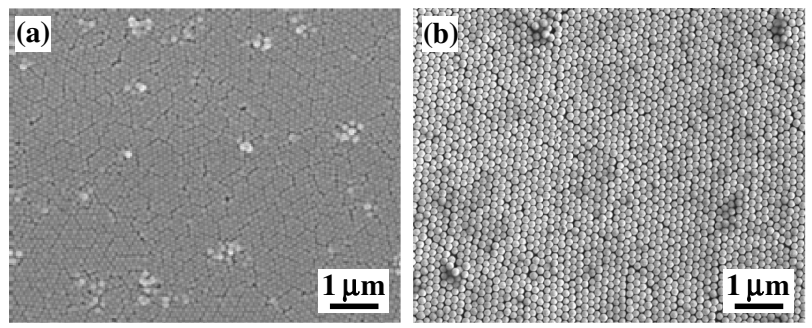

Fig. 3. Typical top-view SEM images of the close-packed colloidal monolayers of (a) $110 \mathrm{~nm}$ and (b) $210 \mathrm{~nm}$ silica spheres as shown in Fig. 2 . with the estimation using the Fresnel equations [3]. The reflectance of the $110 \mathrm{~nm}$ silica coating is apparently much lower than that of the flat control sample, especially for most of the visible wavelengths. This could explain the good antiglare property of the $110 \mathrm{~nm}$ sample shown in Fig. 2(a). By contrast, the assembled $210 \mathrm{~nm}$ silica monolayer shows higher reflectance than that of the flat glass substrate for a wide range of visible wavelengths, matching with the experimental observation in Fig. 2(b). This higher visible reflectance can be attributed to the optical diffraction caused by the self-assembled two-dimensional photonic crystal [20]. However, the $210 \mathrm{~nm}$ sample exhibits lower reflectance than that of the $110 \mathrm{~nm}$ silica coating for a broad range of near-IR bands. The total hemispherical reflectance spectra [Fig. 4(b)] obtained using an Ocean Optics ISP-REF reflectance integrating sphere are quite similar to the above normalincidence spectra. Besides optical reflection measurements, normal-incidence transmission spectra [Fig. 4(c)] were also obtained using the same spectrometer. The $110 \mathrm{~nm}$ sample shows consistently higher transmittance ( $>98 \%$ for a broad band of visible light) than that of the flat glass substrate, while the $210 \mathrm{~nm}$ coating exhibits lower transmittance than that of the flat sample.

The above optical measurements were complemented by numerical simulations using a commercial software Lumerical, which implements the finite-difference timedomain (FDTD) algorithm [21], and a rigorous coupledwave analysis (RCWA) model [22]. The assembled nanoparticles were assumed to be perfectly hexagonally close packed on both sides of a glass substrate. Figure 4(a) compares the FDTD-simulated and experimental specular reflection spectra for colloidal monolayers consisting of 110 and $210 \mathrm{~nm}$ silica spheres. It is clearly evident that the simulations match well with the experiments regarding the shape and peak (and/or valley) position of the reflection spectra. The intrinsic (a)
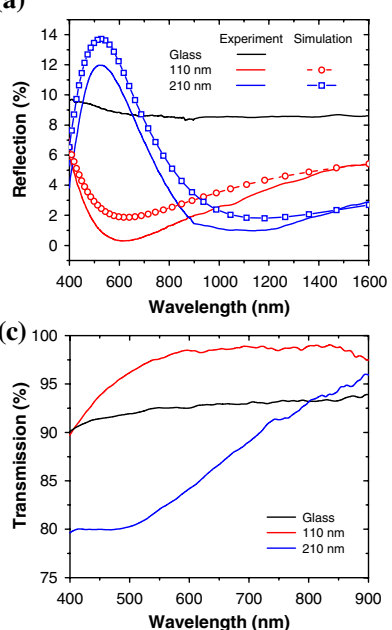

(b)

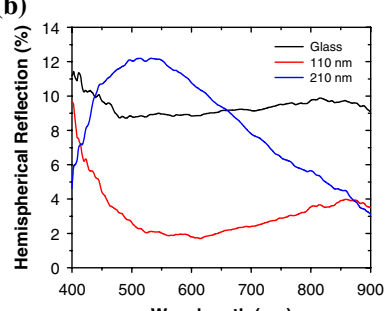

(d)

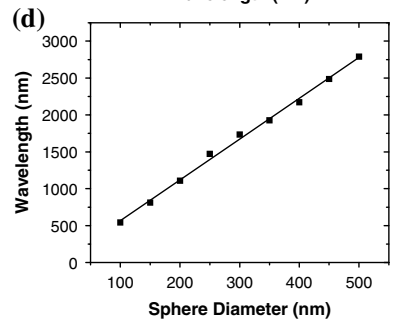

Fig. 4. (Color online) (a) Experimental and FDTD-simulated normal-incidence optical reflection, (b) hemispherical reflection, (c) normal-incidence transmission spectra obtained from a flat glass slide and glass slides covered with close-packed colloidal monolayers of 110 and $210 \mathrm{~nm}$ silica spheres, and (d) FDTD-simulated wavelengths of minimum reflection versus sphere diameters of self-assembled colloidal monolayers. 


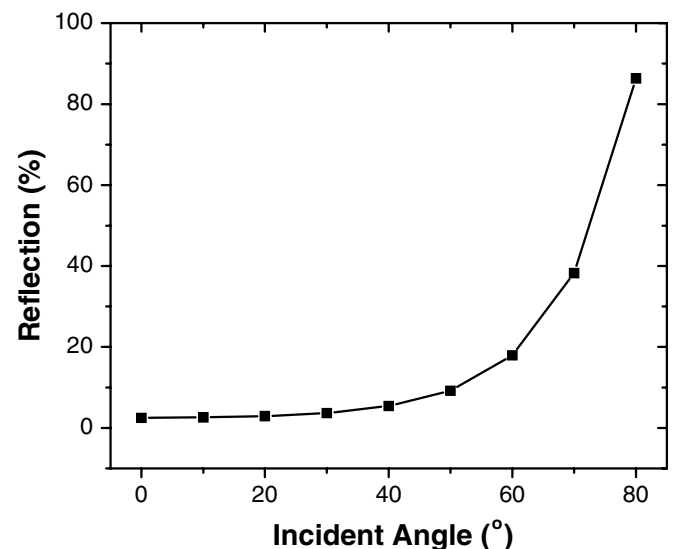

Fig. 5. RCWA-simulated angle-dependent reflection of $600 \mathrm{~nm}$ light from a self-assembled $110 \mathrm{~nm}$ silica monolayer.

defects and the polycrystalline structure of the selfassembled colloidal monolayers (see Fig. 3) could contribute to the small discrepancy in the spectral amplitude between the experimental and simulated spectra. As shown in Fig. 4(a), both experimental and theoretical results indicate that the reflection minimum is a function of particle size. The FDTD-simulated results in Fig. 4(d) illustrate a nearly linear relationship between the reflection minimum and the sphere diameter, which can be explained by using the quarter-wavelength condition for predicting the ideal thickness $\left(h_{c}\right)$ of an antireflection coating: $\lambda_{0}=4 n_{c} h_{c}$, where $\lambda_{0}$ is the wavelength at which destructive interference occurs and $n_{c}$ is the effective refractive index of the coating [3].

The RCWA model generates almost identical simulation results (not shown here). As the RCWA model is less computationally expensive than FDTD, we have therefore utilized it in simulating the angle-dependent reflection from the self-assembled colloidal monolayers. Figure 5 shows the calculated reflectance of $600 \mathrm{~nm}$ light [close to the reflection minimum in Fig. 4(a)] from a $110 \mathrm{~nm}$ silica monolayer at different incident angles. It is apparent that low reflectance $(<5 \%)$ can be achieved for incident angles smaller than $\sim 40^{\circ}$ from the normal, while the reflectance significantly increases for larger incident angles.

In summary, we have developed a simple and inexpensive colloidal self-assembly technology for simultaneously coating both sides of a glass substrate with nanoparticle monolayers as antiglare coatings. As demonstrated in our recent work, this bottom-up approach can be easily employed to create high-quality monolayer coatings on nonplanar optical surfaces over wafer-scale areas [17].

This work was supported by the National Science Foundation under grants CBET-0744879 and CMMI1000686 .

\section{References}

1. M. Ibn-Elhaj and M. Schadt, Nature 410, 796 (2001).

2. U. Schulz, Appl. Opt. 45, 1608 (2006).

3. H. A. Macleod, Thin-Film Optical Filters, 3rd ed. (Institute of Physics, 2001).

4. A. Gombert, B. Blasi, C. Buhler, P. Nitz, J. Mick, W. Hossfeld, and M. Niggemann, Opt. Eng. 43, 2525 (2004).

5. S. Walheim, E. Schaffer, J. Mlynek, and U. Steiner, Science 283, 520 (1999).

6. J. Hiller, J. D. Mendelsohn, and M. F. Rubner, Nat. Mater. 1, 59 (2002).

7. J. Q. Xi, M. F. Schubert, J. K. Kim, E. F. Schubert, M. F. Chen, S. Y. Lin, W. Liu, and J. A. Smart, Nat. Photonics 1, 176 (2007).

8. P. B. Clapham and M. C. Hutley, Nature 244, 281 (1973).

9. Y. F. Huang, S. Chattopadhyay, Y. J. Jen, C. Y. Peng, T. A. Liu, Y. K. Hsu, C. L. Pan, H. C. Lo, C. H. Hsu, Y. H. Chang, C. S. Lee, K. H. Chen, and L. C. Chen, Nat. Nanotechnol. 2, 770 (2007).

10. B. G. Prevo, E. W. Hon, and O. D. Velev, J. Mater. Chem. 17, 791 (2007).

11. Y. Zhao, J. S. Wang, and G. Z. Mao, Opt. Lett. 30, 1885 (2005).

12. H. Jiang, K. Yu, and Y. C. Wang, Opt. Lett. 32, 575 (2007).

13. B. T. Liu and W. D. Yeh, Thin Solid Films 518, 6015 (2010).

14. D. Lee, M. F. Rubner, and R. E. Cohen, Nano Lett. 6, 2305 (2006).

15. N. C. Linn, C. H. Sun, P. Jiang, and B. Jiang, Appl. Phys. Lett. 91, 101108 (2007).

16. J. Zhu, Z. F. Yu, G. F. Burkhard, C. M. Hsu, S. T. Connor, Y. Q. Xu, Q. Wang, M. McGehee, S. H. Fan, and Y. Cui, Nano Lett. 9, 279 (2009).

17. B. M. Phillips, P. Jiang, and B. Jiang, Appl. Phys. Lett. 99, 191103 (2011).

18. W. Stöber, A. Fink, and E. Bohn, J. Colloid Interface Sci. 26, 62 (1968).

19. A. S. Dimitrov and K. Nagayama, Langmuir 12, 1303 (1996).

20. T. A. Birks, P. J. Roberts, P. S. J. Russel, D. M. Atkin, and T. J. Shepherd, Electron. Lett. 31, 1941 (1995).

21. J. Jin, The Finite Element Method in Electromagnetics, 2nd ed. (Wiley, 2002).

22. W. L. Min, B. Jiang, and P. Jiang, Adv. Mater. 20, 3914 (2008). 